\title{
Laboratory validation of a lateral flow device for the detection of CyHV-3 antigens in gill swabs
}

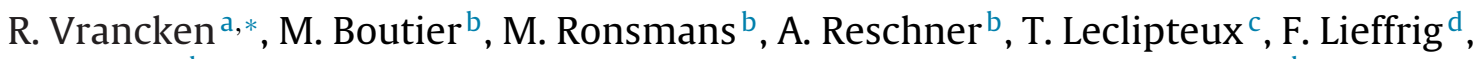 \\ A. Collard ${ }^{\mathrm{d}}$, C. Mélard ${ }^{\mathrm{e}}, \mathrm{S}$. Wera ${ }^{\mathrm{a}}$, J. Neyts ${ }^{\mathrm{a}}, \mathrm{N}$. Goris ${ }^{\mathrm{a}}$, A. Vanderplasschen ${ }^{\mathrm{b}}$ \\ a Okapi Sciences NV, B-3001 Heverlee, Belgium \\ ${ }^{\mathrm{b}}$ University of Liège, Immunology-Vaccinology, Department of Infectious and Parasitic Diseases (B43b), B-4000 Liège, Belgium \\ c Coris BioConcept SPRL, B-5032 Gembloux, Belgium \\ d CER Groupe, B-6900 Marloie, Belgium \\ e EFRA-University of Liège, 10 Chemin de la Justice, B-4500 Tihange, Belgium
}

\section{Article history:}

Received 4 June 2013

Received in revised form 17 July 2013

Accepted 22 July 2013

Available online 26 July 2013

\section{Keywords:}

Cyprinid herpesvirus-3

Koi herpesvirus disease

Lateral flow device

Immunochromatographic assay

\begin{abstract}
A B S T R A C T
Cyprinid herpesvirus-3 (CyHV-3) induces the highly contagious koi herpesvirus disease (KHVD) and may result in significant economic losses to the ornamental and food-producing carp industry. Suspicion of KHVD is triggered by clinical signs and confirmed using laboratory techniques. The latter are labour- and time-consuming, require specialised equipment and trained personnel. For rapid, on-site detection of CyHV-3, a lateral flow device (LFD) was developed using two monoclonal antibodies directed towards the viral glycoprotein ORF65. The LFD was highly specific with analytical and diagnostic specificities of $100 \%$. Analytical sensitivity ranged between $1.25 \times 10^{2}$ and $2.40 \times 10^{4}$ plaque forming units per $\mathrm{ml}$ for isolates originating from geographically distinct regions. In experimentally infected carp, CyHV-3 was detected as early as $4-5$ days post infection. Diagnostic sensitivities of $52.6 \%$ and $72.2 \%$ relative to PCR were recorded, depending on the viral isolate used. When onset of mortality was taken as reference, diagnostic sensitivities increased to $67.0 \%$ and $93.3 \%$. The diagnostic sensitivity for freshly found-dead animals was $100 \%$, irrespective of the virus isolate used. Given the high specificity and ease-of-use for on-site detection of CyHV-3, the LFD was regarded fit for purpose as a first-line diagnostic tool for the identification of acute CyHV-3 infections in KHVD affected (koi) carp.
\end{abstract}

(C) 2013 Elsevier B.V. All rights reserved.
Cyprinid herpesvirus-3 (CyHV-3, also known as koi herpesvirus or KHV) is the causative agent of koi herpesvirus disease (KHVD), a highly contagious disease of common carp (Cyprinus carpio) and its colourful ornamental varieties (koi) (Waltzek et al., 2005). KHVD may result in mortality rates as high as $70-100 \%$. The disease was first reported in Israel in the late 1990s; then rapidly spread throughout the world (Michel et al., 2010a). This rapid spreading was attributed to international fish trade and to koi shows organised around the world. Outbreaks of KHVD are often associated with considerable economic damage to both the food-producing and the ornamental carp industry (Haenen et al., 2004). In order to adequately control KHVD outbreaks, rapid diagnosis is crucial. In general, suspicion of CyHV-3 infection in naive carp populations is triggered by the observation of clinical signs of disease. Even though KHVD does not induce pathognomonic signs, lethargy, overproduction of mucus, discolouration of the gills, and herpetic lesions of the skin have often been associated with CyHV-3 infection.

\footnotetext{
* Corresponding author. Tel.: +32 16299726; fax: +32 16299727.

E-mail address: Robert.vrancken@okapi-sciences.com (R. Vrancken).
}

Neurological signs such as disorientation and loss of equilibrium may be observed at a later stage (OIE, 2012).

Diagnosis of CyHV-3 mainly relies on laboratory techniques, whereby the virus is detected either directly (e.g. CyHV-3 isolation on susceptible cell cultures or PCR-based techniques) or indirectly (detection of virus-induced antibodies by ELISA or neutralisation assays). Particularly, highly sensitive PCR-based techniques have been suggested as the gold standard for CyHV-3 diagnosis (Bergmann et al., 2010), although the World Organisation for Animal Health (OIE) recommends a combination of detection methods (OIE, 2012). A common drawback of all laboratory techniques is that they are labour- and time-consuming, require specialised equipment and trained personnel. A rapid assay, suitable for use under field conditions, could thus be a valuable tool for carp and koi breeders as well as veterinarians in diagnosing CyHV-3 "on-site". The present study aimed at developing and validating an immunochromatographic test for the specific detection of CyHV-3 antigen using a non-invasive approach.

A characterised panel of monoclonal antibodies (mAbs), originating from mice immunised with CyHV-3, were screened by ELISA for reactivity against CyHV-3. Two highly reactive $\mathrm{mAbs}$, hereafter 

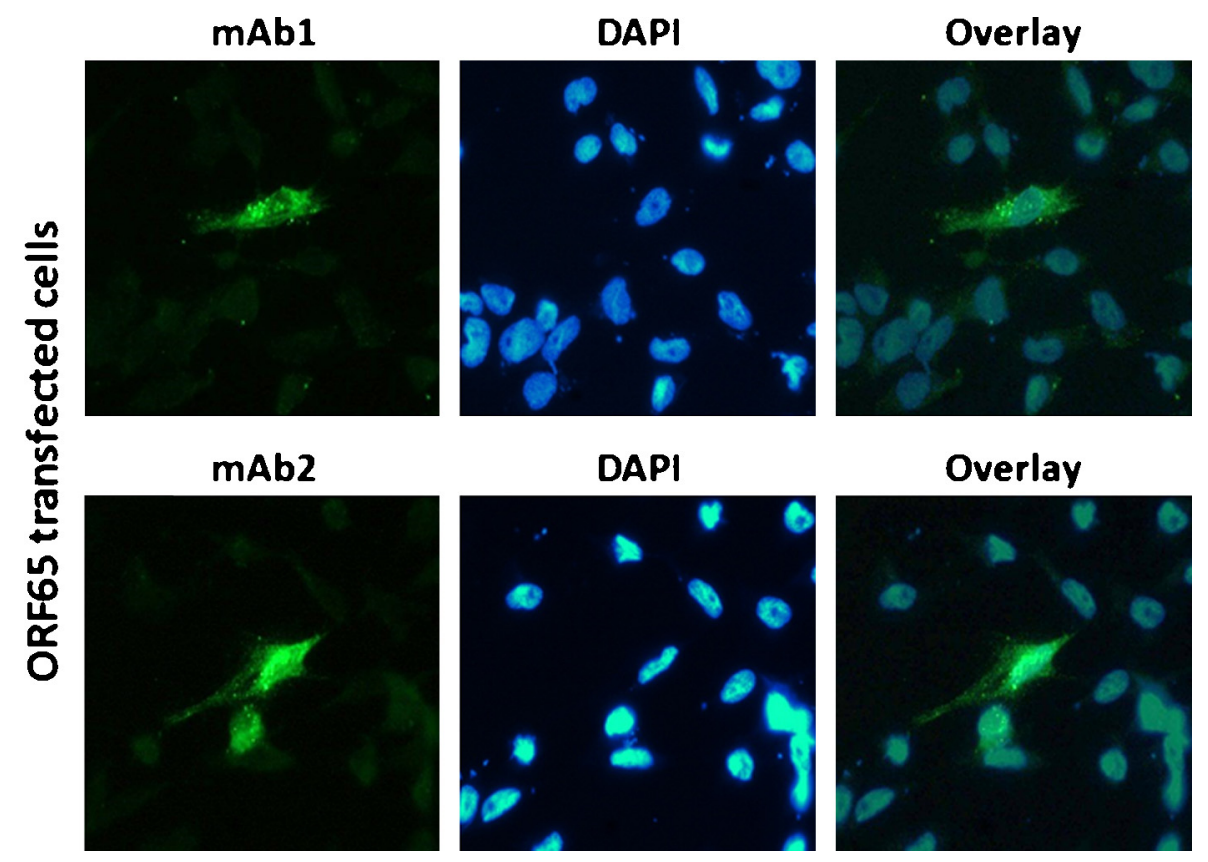

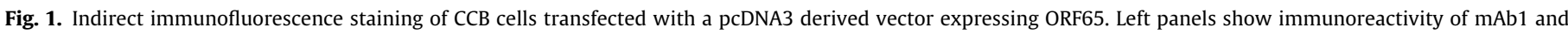

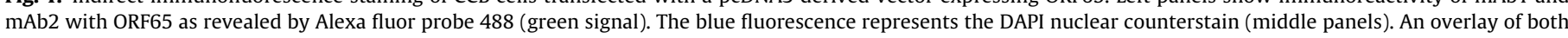
fluorescent signals is depicted in the right panels.

called mAb1 and mAb2, both specific for the structural ORF65 protein (Michel et al., 2010b) were selected for incorporation into a lateral flow device (LFD). The molecular target of these mAbs was verified by indirect immunofluorescence staining of common carp brain cells $(\mathrm{CCB})$ transfected with pcDNA3 derived vector expressing CyHV-3 ORF65 (Invitrogen). Cells were transfected using the Lipofectamine Plus reagent (Invitrogen). Transfected cells were stained using either mAb1 or 2 as primary antibody and an Alexa Fluor 488-conjugated goat anti-mouse immunoglobulin $\mathrm{G}$ as the second conjugate as published earlier (Costes et al., 2008). In parallel, a nuclear counterstain (DAPI) was performed according to the manufacturer's protocol (Invitrogen). Microscopic analysis, carried out as described earlier (Costes et al., 2008), revealed a positive immunoreactivity of mAb1 and mAb2 with ORF65 (Fig. 1).

Using a proprietary method (Coris BioConcept SPRL), mAb1 was coupled to colloidal gold particles, adsorbed on a conjugate pad and applied to the base of a plastic-backed nitrocellulose membrane. The $\mathrm{mAb} 2$ was immobilised on the nitrocellulose membrane at the test line $(\mathrm{T}$ ) and a control antibody (in casu a goat anti-mouse antibody) was immobilised at the control line (C). The LFD was assembled by embedding the test strip in a plastic cassette (see Fig. 2A).

To determine the LFD's analytical sensitivity, two-fold serial dilution series of in vitro cultured isolates of CyHV-3 were eluted in $250 \mu \mathrm{l}$ of a proprietary elution buffer (Coris BioConcept SPRL). The test was carried out by transferring $100 \mu$ l of eluent to the sample pad of the LFD. Once applied, the gold-conjugated mAb1 is reconstituted in the conjugate pad. When CyHV-3 antigen is present in the eluent, an antigen/mAb1 complex is formed. This complex migrates through the nitrocellulose membrane by capillary force and encounters the immobilised $\mathrm{mAb} 2$ at $T$. Consequently, the antigen/mAb1 complex is captured at $T$ and a reddish-brown colour appears (Fig. 2B). Remnants of the conjugated complex migrate further and are captured by the goat anti-mouse control antibody resulting in a similar signal at $C$, substantiating the test result (Fig. 2B). Results of the test are read 15 min following application of the eluent to the sample pad. The analytical sensitivity was evaluated with four CyHV-3 strains originating from three countries.
The FL (Costes et al., 2008) and the M3 strains were isolated from the kidney of carp that died of KHVD in two distinct geographical regions of Belgium. A German isolate $(G)$ (kindly provided by Dr. Fuchs, FLI, Germany) and an Israeli isolate (I) (kindly provided by Dr. Kotler, The Hebrew University Hadassah Medical School, Israel) were also used. All CyHV-3 strains were produced on CCB cells. Clarified cell supernatants were stored at $-80^{\circ} \mathrm{C}$ and titrated before use. The LFD was able to detect all tested isolates with an analytical sensitivity ranging from $1.25 \times 10^{2}$ to $2.40 \times 10^{4}$ plaque forming units per $\mathrm{ml}$ (pfu/ml) (Table 1$)$.

The analytical specificity of the LFD was evaluated using other known viral pathogens of carp, namely Spring viraemia of carp virus (SVCV; kindly provided by Dr. Bremont, INRA, France) and Cyprinid herpesvirus-1 (CyHV-1; UKG364 P2 isolate kindly provided by Dr. Keith Way, CEFAS, UK). SVCV and CyHV-1 were produced on Epithelioma Papulosum Cyprini (EPC) cells and KF-1 (koi fin) cells, respectively. Clarified cell supernatants were stored at $-80^{\circ} \mathrm{C}$ and titrated before use. SVCV was chosen because this carp pathogen, like CyHV-3, can induce mortality without associated pathognomonic clinical signs; whereas CyHV-1 is genetically the most closely-related aquatic pathogen to CyHV-3 able to infect common carp. No cross-reactivity or false positive results were observed on the LFD when the analyses where performed on a twofold dilution series in elution buffer of the virus stock of SVCV $\left(3.5 \times 10^{8} \mathrm{pfu} / \mathrm{ml}\right)$ and CyHV-1 $\left(2.4 \times 10^{6} \mathrm{pfu} / \mathrm{ml}\right)$; indicating an analytical specificity of $100 \%$ for the LFD and ensuring the correct diagnosis of KHVD in case of a positive LFD result.

To define the diagnostic sensitivity of the LFD, two identical experimental infection studies in carp were carried out using

Table 1

Analytical sensitivity of the LFD on CyHV-3 isolates from different geographical regions.

\begin{tabular}{lll}
\hline CyHV-3 isolate & Origin & Detection limit $(\mathrm{pfu} / \mathrm{ml})$ \\
\hline I & Israel & $1.5 \times 10^{3}$ \\
FL & Belgium & $2.4 \times 10^{4}$ \\
M3 & Belgium & $3.1 \times 10^{3}$ \\
G & Germany & $1.25 \times 10^{2}$ \\
\hline
\end{tabular}




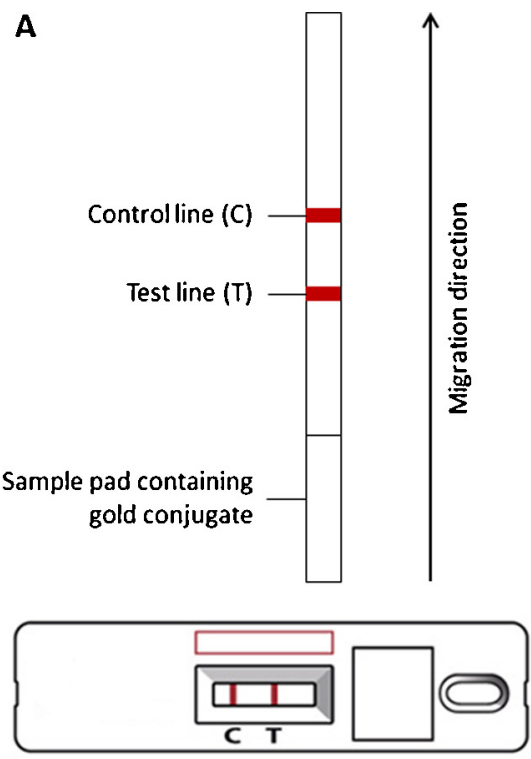

B
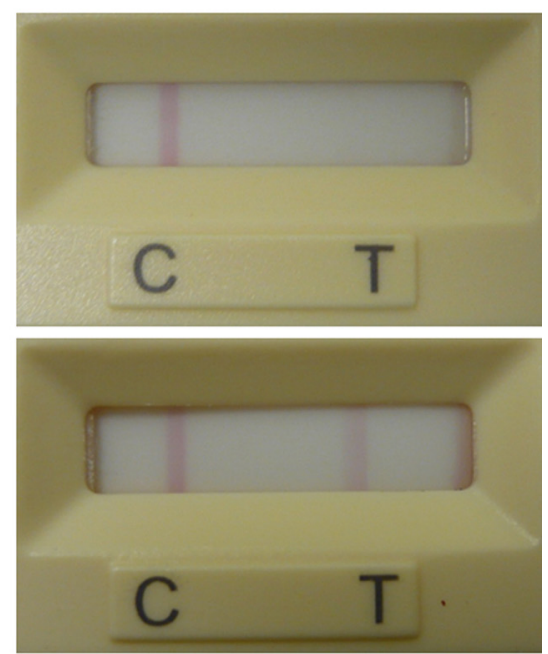

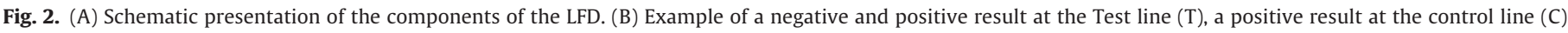
validates the obtained result.

the Belgian field isolates CyHV-3 FL or CyHV-3 M3 as inoculum. This animal study was accredited by the local ethics committee of the University of Liège, Belgium (Laboratory accreditation No.1610008). For each experiment, 120 common carp (average bodyweight of approximately $10 \mathrm{~g}$ ) were distributed over four 60litre tanks. Each tank was equipped with an individual recirculating water system and kept at the permissive temperature of $25^{\circ} \mathrm{C}$. After a 5-day acclimation period, three tanks containing 30 carp each were infected by bath infection for $1 \mathrm{~h}$ with CyHV-3 at an inoculation dose of $40 \mathrm{pfu} / \mathrm{ml}$. One tank holding 30 carp served as negative control. Daily, for a period of 10 consecutive days post infection (p.i.) and every other day from day 10 to 16 p.i., two animals per tank were randomly selected (they were not put back in the tank to avoid introducing bias as a consequence of repeated handling). Gills of fish selected at random were swabbed and tested for the presence of CyHV-3 antigen using the LFD. All animals found dead during the experiment were removed, the gills were swabbed and tested as well. For comparison, gill tissue samples of dead animals selected at random were also tested in a CyHV-3 specific polymerase chain reaction (PCR) assay based on a published protocol (Bercovier et al., 2005) which is one of two PCR assays described in the OIE Manual of Diagnostic Tests for Aquatic Animals (OIE, 2012). To this end, $50 \mathrm{mg}$ of gill tissue was homogenised mechanically in $200 \mu \mathrm{l}$ of phosphate buffered saline using the Precellys ${ }^{\circledR} 24$ tissue homogeniser according to the manufacturer's instructions (Precellys, France). Subsequently, total DNA was extracted from $100 \mu \mathrm{l}$ of homogenate using the QIAamp DNA Mini Kit (Qiagen, France). Extracted DNA was eluted in $50 \mu \mathrm{l}$ of RNase/DNase-free water of which $2.5 \mu$ l was used for PCR analysis.

When the CyHV-3 isolate FL was used for inoculation, the LFD detected viral antigen in 2 out of 6 carp (i.e. two randomly selected carp for each of the three tanks) at day 5 p.i. Hence, a detection rate of 33.3\% was observed. Similarly, at day 6 p.i., viral antigen on the LFD was detected in 2 out of 6 carp. The detection rate steadily increased to $100 \%$ on day 9 p.i. and subsequently decreased until the end of the observation period corresponding to day 16 p.i. (Fig. 3). Mortality due to KHVD was first observed on day 7 p.i. All carp found dead $(n=16)$ were positive for CyHV-3 antigen on the LFD. In parallel, the presence of viral DNA was evaluated by means of PCR. At day 3 p.i., 5 out of 6 animals tested positive on PCR. Thereafter, all animals were found to be positive for CyHV-3 DNA until the end of the experiment (day 16 p.i.). Consequently, the LFD displayed a diagnostic sensitivity of $52.6 \%$ relative to PCR.

When using the CyHV-3 isolate M3 for inoculation, the LFD detected viral antigen in 2 out of 6 animals at day 4 p.i. (detection rate equalled 33.3\%) and in 5 out of 9 animals at day 5 p.i. (2 out of 6 randomly selected animals and 3 out of 3 animals found dead; equalling a detection rate of 55.6\%). The detection rate rapidly increased to $91.7 \%$ at day 6 p.i. and $100 \%$ at day 7 and 8 p.i. The detection rate subsequently decreased to $88.9 \%$ at day 9 p.i. and the experiment was halted because all animals were found dead (Fig. 3). Mortality due to KHVD started from day 5 p.i. and all animals found dead $(n=32)$ tested positive on the LFD. In parallel, 1 out of 6 animals tested PCR positive on day 1 p.i., and 5 out of 6 animals were positive for viral DNA on day 2 p.i. Thereafter, all carp tested PCR positive for the remainder of the experiment until 9 days p.i. Hence, the LFD exhibited a diagnostic sensitivity of $72.2 \%$ relative to PCR.

The diagnostic specificity of the LFD test was evaluated by testing gill swabs of 60 common carp, known to be negative for CyHV-3 (originating from the two control tanks). No (false) positive signals were observed, indicating a diagnostic specificity of $100 \%$ for the LFD.

The current study presents the first rapid immunochromatographic test for the specific detection of CyHV-3-antigen. Criteria such as ease of sampling (including accessibility of sampling site), absence of background signal on the LFD, and intensity of the signal read-out were taken into account when deciding on the sampling site (swab sites tested: eye, gill, skin with and without mucus, faecal matter, intestinal tract, kidney). This resulted in the selection of gill swabs as the preferred sampling site. A non-invasive sampling procedure, thereby preventing unnecessary sacrifice of the animal, was opted for since carp with unknown disease status may suffer from untreatable as well as treatable diseases. A non-invasive sampling procedure is of particular importance to recreational koi-keepers, owning frequently ornamental koi of substantial value. Furthermore, the LFD was intended to identify CyHV-3 in the acute phase of the infection as a first-line diagnostic tool, and CyHV-3 is known to reach the highest levels in the gills at permissive water temperatures (Gilad et al., 2004). Although the analytical and diagnostic specificities of the LFD equal $100 \%$, the relative diagnostic sensitivity of the LFD is inferior to that of the PCR assay. Nevertheless, the 


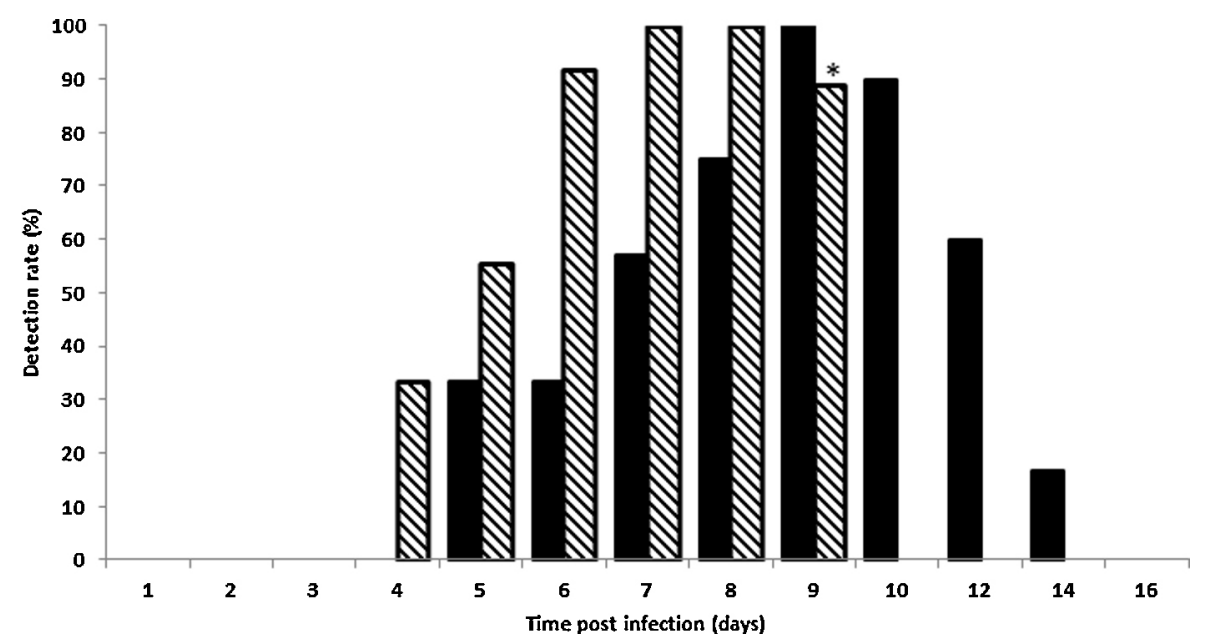

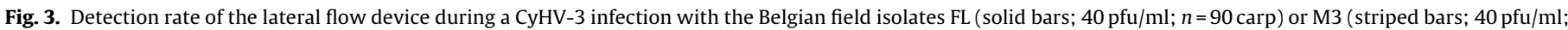

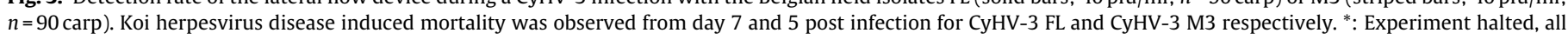
remaining carp were found dead.

latter does not invalidate the use of this LFD for the following reasons. Carp keepers can roughly be divided into (i) breeders, where large numbers of carp are present (breeders of carp for human consumption or koi, ponds for recreational fishing) and (ii) hobbyists or recreational keepers, owning small(er) numbers of animals.

When large volumes of carp are housed together, KHVD diagnosis in the early stage of infection based on clinical signs is difficult and generally, suspicion is triggered only when increased mortality is observed in the carp population. When onset of mortality is regarded as the primary criterion for raising KHVD suspicion, the diagnostic sensitivity of the LFD increased to $67.0 \%$ (for the moderately virulent CyHV-3 FL strain) and 93.3\% (for the highly virulent CyHV-3 M3 strain). Moreover, the LFD test has a detection rate of $100 \%$ in newly found-dead carp. Hence, CyHV-3 diagnosis will most likely be reached under these (field) circumstances. Hobbyists are often equipped with basic material for the diagnosis of parasitic and bacterial infestations and are known to monitor carefully the individual carp's health status. It is important to note that in the present study, the diagnostic sensitivity of the LFD was determined on randomly selected fish and not on fish displaying the fastest progression of the disease. KHVD symptoms increase with time post-infection as the viral charges in the organs (Gilad et al., 2004). Consequently, it is likely that the sensitivity of the test performed by hobbyists on sick fish will be higher than the one determined under the present experimental conditions.

For both professional breeders and hobbyists, a rapid and highly specific test such as the LFD could be an easy "on-site" diagnostic tool. A read-out of the test result is obtained within $15 \mathrm{~min}$ after sampling without the need of sophisticated equipment or trained personnel. This is in contrast to laboratory techniques, including PCR, which are relatively expensive, time- and labour-consuming requiring specialised equipment, trained personnel and the shipment of samples to a diagnostic laboratory.

Taking these considerations and the test characteristics into account, the LFD is fit for purpose as a rapid, highly specific first-line diagnostic tool for the identification of acute CyHV-3 infections in KHVD affected (koi) carp.

\section{Conflict of interest}

Robert Vrancken, Nesya Goris, Stefaan Wera and Johan Neyts have a financial interest in Okapi Sciences NV. Thierry Leclipteux has a financial interest in Coris BioConcept SPRL.

\section{Acknowledgments}

This study was funded by the Walloon Region (APE grant MA13387-00). Alain Vanderplasschen is a member of the BELVIR consortium (IAP, phase VII) granted by the Belgian Science Policy Office (BELSPO) (Belgium). A. Vanderplasschen and C. Mélard are members of the AFFISH research centre of the University of Liège.

\section{References}

Bercovier, H., Fishman, Y., Nahary, R., Sinai, S., Zlotkin, A., Eyngor, M., Gilad, O., Eldar A., Hedrick, R.P., 2005. Cloning of the koi herpesvirus (KHV) gene encoding thymidine kinase and its use for a highly sensitive PCR based diagnosis. BMC Microbiol. 5, 13.

Bergmann, S.M., Riechardt, M., Fichtner, D., Lee, P., Kempter, J., 2010. Investigation on the diagnostic sensitivity of molecular tools used for detection of koi herpesvirus. J. Virol. Methods 163, 229-233.

Costes, B., Fournier, G., Michel, B., Delforge, C., Raj, V.S., Dewals, B., Gillet, L., Drion, P., Body, A., Schynts, F., Lieffrig, F., Vanderplasschen, A., 2008. Cloning of the koi herpesvirus genome as an infectious bacterial artificial chromosome demonstrates that disruption of the thymidine kinase locus induces partial attenuation in Cyprinus carpio koi. J. Virol. 82, 4955-4964.

Gilad, O., Yun, S., Zagmutt-Vergara, F.J., Leutenegger, C.M., Bercovier, H., Hedrick, R.P., 2004. Concentrations of a Koi herpesvirus (KHV) in tissues of experimentally infected Cyprinus carpio koi as assessed by real-time TaqMan PCR. Dis. Aquat. Organ. 60, 179-187.

Haenen, O.L.M., Way, K., Bergmann, S.M., Ariel, E., 2004. The emergence of koi herpesvirus and its significance to European aquaculture. Bull. Eur. Assoc. Fish Pathol. 24, 293-307.

Michel, B., Fournier, G., Lieffrig, F., Costes, B., Vanderplasschen, A., 2010a. Cyprinid herpesvirus 3. Emerg. Infect. Dis. 16, 1835-1843.

Michel, B., Leroy, B., Stalin Raj, V., Lieffrig, F., Mast, J., Wattiez, R., Vanderplasschen, A.F., Costes, B., 2010b. The genome of cyprinid herpesvirus 3 encodes 40 proteins incorporated in mature virions. J. Gen. Virol. 91, 452-462.

OIE, 2012. Manual of Diagnostic Tests for Aquatic Animals.

Waltzek, T.B., Kelley, G.O., Stone, D.M., Way, K., Hanson, L., Fukuda, H., Hirono, I., Aoki, T., Davison, A.J., Hedrick, R.P., 2005. Koi herpesvirus represents a third cyprinid herpesvirus (CyHV-3) in the family Herpesviridae. J. Gen. Virol. 86, 1659-1667. 